Case Report

\title{
A Rare Case of Takotsubo Syndrome and Acute Coronary Syndrome of the Right Coronary Artery
}

\author{
Nicholas Mencer $\mathbb{D}^{1},{ }^{1}$ Larry Todd Justice, ${ }^{1}$ William Black, ${ }^{2}$ and Kayleigh Litton $\mathbb{D}^{2}$ \\ ${ }^{1}$ Department of Interventional Cardiology Methodist Medical Center, Oak Ridge, Tennessee 37748, USA \\ ${ }^{2}$ Department of Interventional Cardiology, The University of Tennessee Knoxville, Knoxville, Tennessee 37996, USA \\ Correspondence should be addressed to Nicholas Mencer; nicholas.mencer@lmunet.edu
}

Received 18 February 2019; Revised 24 April 2019; Accepted 12 May 2019; Published 9 June 2019

Academic Editor: Assad Movahed

Copyright (c) 2019 Nicholas Mencer et al. This is an open access article distributed under the Creative Commons Attribution License, which permits unrestricted use, distribution, and reproduction in any medium, provided the original work is properly cited.

\begin{abstract}
Takotsubo syndrome (TTS) is an increasingly recognized heart disease that was initially regarded as a benign condition, but since has proven to cause irreversible myocardial damage, resembling that of acute coronary syndrome (ACS). The etiology of TTS is still uncertain but may be associated with catecholamine elevations during times of emotional or physical stress. Catecholamines are also understood to have prothrombotic properties, which could lead to ACS. With these similarities, differentiating these two pathologies can be difficult, especially when TTS and ACS occur simultaneously.
\end{abstract}

\section{Introduction}

The pathogenesis of takotsubo syndrome (TTS) is not completely understood. Proposed mechanisms involve catecholamine excess during times of physical or emotional stress that lead to left ventricular myocardial dysfunction by direct catecholamine-associated toxicity and microvascular spasm $[1,2]$. Although TTS may sometimes present as an ST elevation myocardial infarction (STEMI), angiography typically reveals no occlusive lesion within the coronary arteries. We present a case of a postmenopausal Caucasian female with TTS and coexisting critical stenosis of the right coronary artery (RCA).

\section{Case Report}

A previously healthy 89-year-old female presented to the emergency department complaining of midsternal chest discomfort that radiated to her back. Her chest discomfort began the day prior to presentation, but she initially attributed it to indigestion and thus waited to seek medical attention. Her chest pain persisted however, which prompted her to seek evaluation in the emergency department. Upon arrival, she was given aspirin with resolution of her symp- toms. Laboratory analysis revealed an elevated troponin I level of 0.319 and initial creatine kinase (CK) of 12.7. Brain natriuretic peptide level on presentation was not checked. Electrocardiogram (ECG) was notable for diffuse T-wave inversions demonstrating inferior, as well as anterolateral ischemia, and a prolonged QTc of $503 \mathrm{~ms}$ (Figure 1). Echocardiogram demonstrated moderate left ventricular dysfunction (ejection fraction of $35 \%-40 \%$ ) with mid to distal anteroseptal, anterolateral, and apical akinesis (Figure 2). The patient was taken to the catheterization suite and underwent emergent left heart catheterization. Angiography revealed $90 \%$ stenosis in the mid right coronary artery (RCA) which was believed to be the culprit lesion. There were no significant obstructive lesions noted in the left anterior descending or left circumflex arteries. She underwent percutaneous coronary intervention (PCI) with successful stent placement to the RCA (Figure 3). She was then started on appropriate guideline-directed medical therapy and observed in the intensive care unit where her chest pain resolved. Repeat laboratory analysis revealed that $\mathrm{CK}$ had decreased to 6.4. ECG obtained following PCI revealed interval improvement of the inferior T-wave inversions, with sustained $\mathrm{T}$-wave abnormalities in the anterolateral leads (Figure 4). Cardiac magnetic resonance imaging is 




FIgure 1: ECG on admission demonstrating diffuse T-wave inversions.

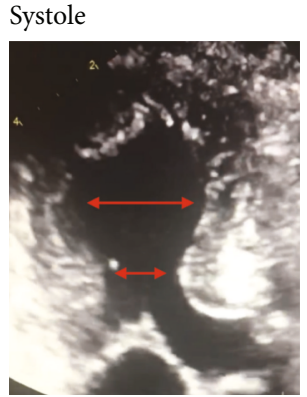

(a)



(b)
Figure 2: Apical three chamber echocardiogram demonstrating left ventricular apical ballooning and basal hyperkinesis consistent with takotsubo syndrome.

not available at our facility and was not performed. The remainder of her hospitalization proceeded without incident, and she was discharged home in stable condition three days later.

The patient was evaluated two weeks after discharge in an outpatient clinic and was feeling well. She again denied any stressors prior to the onset of pain but reported that the development of chest pain caused her a great deal of emotional distress. Repeat echocardiogram was obtained which revealed normal left ventricular systolic function (Figure 5).

\section{Discussion}

There have been numerous case reports of patients presenting with profound left ventricular dysfunction after suffering severe emotional or physical stress. This syndrome was first described in Japan in 1990 and is commonly referred to as transient apical ballooning syndrome or takotsubo syndrome [3-8]. Takotsubo syndrome is often associated with emotional triggers but has frequently been described in association with physical triggers. A large registry of patients with TTS found that physical triggers were more common than emotional triggers-women were more likely to have emotional triggers whereas men were more likely to have a physical trigger [9]. Although the diagnosis of TTS has historically been based on the presence of angiographically normal coronary arteries, there have been multiple reports of TTS occurring in the setting of physiological stress from acute coronary syndrome (ACS) [10]. The prevalence of TTS is estimated to be $2 \%-3 \%$ in patients who present with ACS and may be
Right anterior oblique view of the right coronary artery

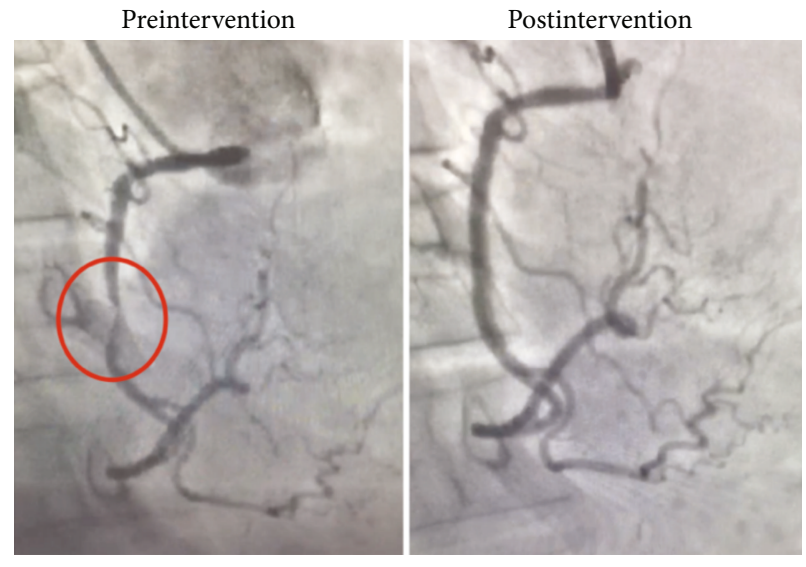

Anterior-posterior cranial and left anterior oblique caudal views of left coronary artery

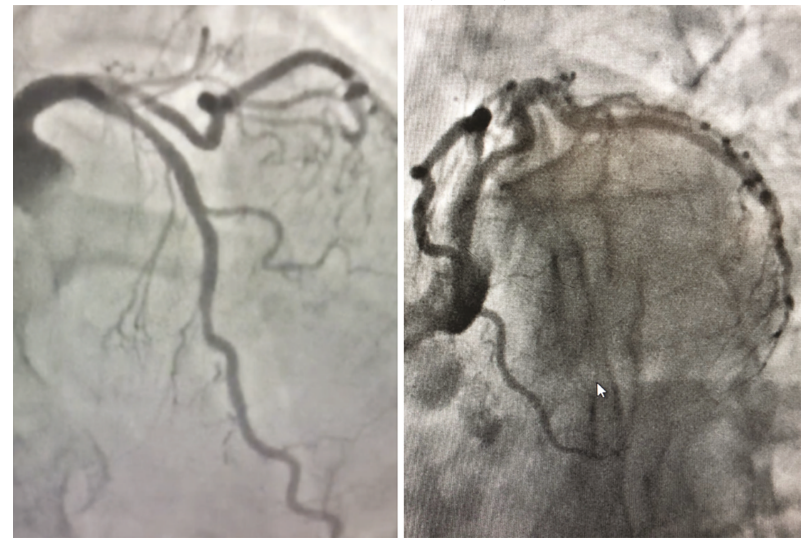

Figure 3: Angiographical images obtained during cardiac catheterization.

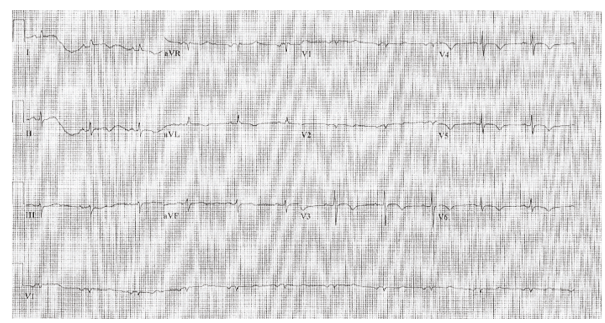

FIGURE 4: ECG post-PCI demonstrating interval improvement of inferior $\mathrm{T}$-wave inversions and sustained $\mathrm{T}$-wave abnormalities in the anterolateral leads.

underestimated in individuals with coexisting coronary artery disease [11]. The recently updated International Takotsubo Diagnostic Criteria (InterTAK Diagnostic Criteria) specifically states that "significant coronary artery disease is not a contraindication in takotsubo syndrome" [1]. The exact pathophysiology of myocardial dysfunction due to sympathetic stimulation is unknown, but numerous mechanisms have been proposed. One potential mechanism is direct myocardial ischemia due to coronary arterial spas$\mathrm{m}$-it has been shown that increased mental stress can cause vasoconstriction in patients without coronary artery disease 


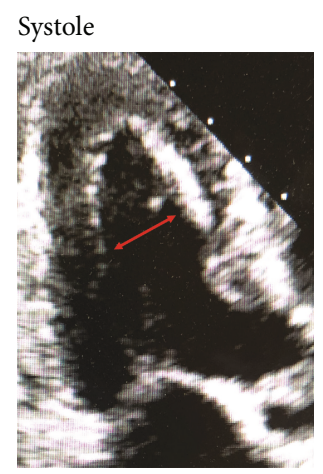

(a)

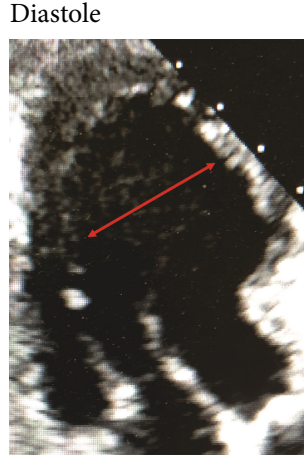

(b)
FIGURE 5: Apical three chamber echocardiogram obtained two weeks following revascularization of the RCA demonstrating no residual left ventricular dysfunction.

[12]. Another potential mechanism is sympathetically mediated microvascular dysfunction resulting in abnormal coronary blood flow in the absence of obstructive disease [13]. A third potential mechanism is direct myocyte injury due to increased levels of circulating catecholamines which have been shown to cause contraction band necrosis. Similar injury patterns have been reported with catecholamine excess due to pheochromocytoma and subarachnoid hemorrhage $[14,15]$.

However, recent review suggests that although catecholamine excess may be linked to TTS, there is no direct causality [16].

This case poses two interesting questions: is the physiologic and emotional stress of chest pain enough to cause TTS? Can the catecholamine excess from TTS cause ACS? Catecholamines have been demonstrated to have prothrombotic properties in coronary arteries. Lin and Young demonstrated that by raising the plasma epinephrine concentration to approximately $27 \mathrm{nmol} / \mathrm{L}$, the incidence of cyclic blood flow reductions in the coronary arteries of canines increased by $60 \%$ [17]. Catecholamine levels in takotsubo patients are 2-3 times higher than myocardial infarction patients 1-2 days after the onset of symptoms and 20 times higher than normal adults [18]. Additionally, the catecholamine levels in takotsubo patients remain elevated over myocardial infarction levels for 7-9 days [19]. With the known prothrombotic properties of catecholamines and the suspected rise in catecholamines associated with TTS, it is possible that TTS could have occurred first in our patient, leading to ACS of the RCA. This could have occurred either by thrombosis in our patient's native coronary artery or with the acute left ventricular (LV) systolic dysfunction associated TTS, an embolic event from a LV thrombus. However, extensive review of our patient's history, cardiac imaging, laboratory findings, and medical record failed to elucidate a possible cause for TTS other than her ACS.

Based on her description of symptoms and failure to identify another cause, we hypothesize that she initially developed ACS of the RCA, and the emotional distress from this event is likely the etiology of her TTS. Previous case series have reported that postischemic myocardial stunning has features typical of TTS and suggested that ACS may trigger TTS [20]. It can be difficult to distinguish between TTS and ACS as they have similar clinical presentations, and both can cause transient wall motion abnormalities [21]. Cardiac magnetic resonance imaging (CMR) can help distinguish TTS from myocardial infarction when there is diagnostic uncertainty, but unfortunately, we were unable to perform this in our patient [22].

\section{Conclusion}

The diagnosis of TTS has historically been a diagnosis of exclusion that required the presence of normal coronary arteries, but recent expert consensus has been updated to remove CAD as an exclusion criterion. Previous case reports have described cases of TTS coexisting with ACS as well as cases of ACS triggering TTS. This case contributes to the growing body of case reports that suggest that TTS and ACS can occur concomitantly.

\section{Disclosure}

Nicholas Mencer, DO, Larry Todd Justice, MD, William Black, MD, and Kayleigh Litton, DO certify that they have no affiliations with or involvement in any organization or entity with any financial interest (such as honoraria; educational grants; participation in speakers' bureaus; membership, employment, consultancies, stock ownership, or other equity interest; and expert testimony or patentlicensing arrangements) or nonfinancial interest (such as personal or professional relationships, affiliations, knowledge, or beliefs) in the subject matter or materials discussed in this manuscript.

\section{Conflicts of Interest}

The authors declare that they have no conflicts of interest.

\section{Authors' Contributions}

All authors had access to all data and a role in writing this manuscript.

\section{References}

[1] J.-R. Ghadri, I. S. Wittstein, A. Prasad et al., "International expert consensus document on takotsubo syndrome (part I): clinical characteristics, diagnostic criteria, and pathophysiology," European Heart Journal, vol. 39, no. 22, pp. 2032-2046, 2018.

[2] H. M. Nef, H. Mollmann, S. Kostin et al., "Tako-Tsubo cardiomyopathy: intraindividual structural analysis in the acute phase and after functional recovery," European Heart Journal, vol. 28, no. 20, pp. 2456-2464, 2007.

[3] D. Pavin, H. Le Breton, and C. Daubert, "Human stress cardiomyopathy mimicking acute myocardial syndrome," Heart, vol. 78, no. 5, pp. 509-511, 1997.

[4] S. Kawai, H. Suzuki, H. Yamaguchi et al., "Ampulla cardiomyopathy ('takotsubo' cardiomyopathy) - reversible left 
ventricular dysfunction: with ST segment elevation," Japanese Circulation Journal, vol. 64, 2000.

[5] R. P. Villareal, A. Achari, S. Wilansky, and J. M. Wilson, "Anteroapical stunning and left ventricular outflow tract obstruction," Mayo Clinic Proceedings, vol. 76, no. 1, pp. 7983, 2001.

[6] H. Z. Brandspiegel, R. A. Marinchak, S. J. Rials, and P. R. Kowey, "A broken heart," Circulation, vol. 98, no. 13, pp. 1349-1349, 1998.

[7] K. Tsuchihashi, K. Ueshima, T. Uchida et al., "Transient left ventricular apical ballooning without coronary artery stenosis: a novel heart syndrome mimicking acute myocardial infarction," Journal of the American College of Cardiology, vol. 38, no. 1, pp. 11-18, 2001.

[8] S. Kurisu, H. Sato, T. Kawagoe et al., "Tako-tsubo-like left ventricular dysfunction with ST-segment elevation: a novel cardiac syndrome mimicking acute myocardial infarction," American Heart Journal, vol. 143, no. 3, pp. 448-455, 2002.

[9] C. Templin, J. R. Ghadri, J. Diekmann et al., "Clinical features and outcomes of takotsubo (stress) cardiomyopathy," The New England Journal of Medicine, vol. 373, no. 10, pp. 929-938, 2015.

[10] S. Y-Hassan, "Acute coronary syndrome or takotsubo syndrome: most probably both of them, the first has triggered the second," International Journal of Cardiology, vol. 190, pp. 367-368, 2015.

[11] K. Kato, A. R. Lyon, J. R. Ghadri, and C. Templin, “Takotsubo syndrome: aetiology, presentation and treatment," Heart, vol. 103, no. 18, pp. 1461-1469, 2017.

[12] C. R. Lacy, R. J. Contrada, M. L. Robbins et al., "Coronary vasoconstriction induced by mental stress (simulated public speaking)," The American Journal of Cardiology, vol. 75, no. 7, pp. 503-505, 1995.

[13] K. A. Bybee, A. Prasad, G. W. Barsness et al., "Clinical characteristics and thrombolysis in myocardial infarction frame counts in women with transient left ventricular apical ballooning syndrome," The American Journal of Cardiology, vol. 94, no. 3, pp. 343-346, 2004.

[14] C. Wilkenfeld, M. Cohen, S. L. Lansman et al., "Heart transplantation for end-stage cardiomyopathy caused by an occult pheochromocytoma," The Journal of Heart and Lung Transplantation, vol. 11, pp. 363-366, 1992.

[15] G. Neil-Dwyer, P. Walter, J. M. Cruickshank, B. Doshi, and P. O'Gorman, "Effect of propranolol and phentolamine on myocardial necrosis after subarachnoid haemorrhage," BMJ, vol. 2, no. 6143, pp. 990-992, 1978.

[16] S. Y-Hassan, "Plasma epinephrine levels and its causal link to takotsubo syndrome revisited: critical review with a diverse conclusion," Cardiovascular Revascularization Medicine, 2018.

[17] H. Lin and D. B. Young, "Opposing effects of plasma epinephrine and norepinephrine on coronary thrombosis in vivo," Circulation, vol. 91, no. 4, pp. 1135-1142, 1995.

[18] I. S. Wittstein, D. R. Thiemann, J. A. C. Lima et al., "Neurohumoral features of myocardial stunning due to sudden emotional stress," The New England Journal of Medicine, vol. 352, no. 6, pp. 539-548, 2005.

[19] E. Kazakauskaitè, A. Jankauskas, T. Lapinskas, R. Ordienė, and E. Ereminienè, "Takotsubo cardiomyopathy: the challenging diagnosis in clinical routine," Medicina, vol. 50, no. 1, pp. 1-7, 2014.
[20] S. Y-Hassan, "Takotsubo syndrome triggered by acute coronary syndrome in a cohort of 20 patients: an often missed diagnosis," International Journal of Cardiology and Research, vol. 2, pp. 28-33, 2015.

[21] L. C. Napp, J. R. Ghadri, V. L. Cammann, J. Bauersachs, and C. Templin, "Takotsubo cardiomyopathy: completely simple but not so easy," International Journal of Cardiology, vol. 197, pp. 257-259, 2015.

[22] J. R. Ghadri, I. S. Wittstein, A. Prasad et al., "International expert consensus document on takotsubo syndrome (Part II): diagnostic workup, outcome, and management," European Heart Journal, vol. 39, no. 22, pp. 2047-2062, 2018. 


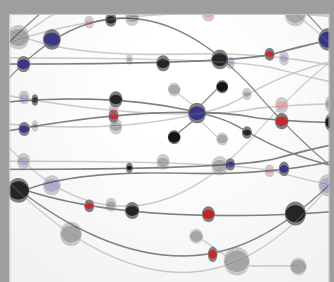

The Scientific World Journal
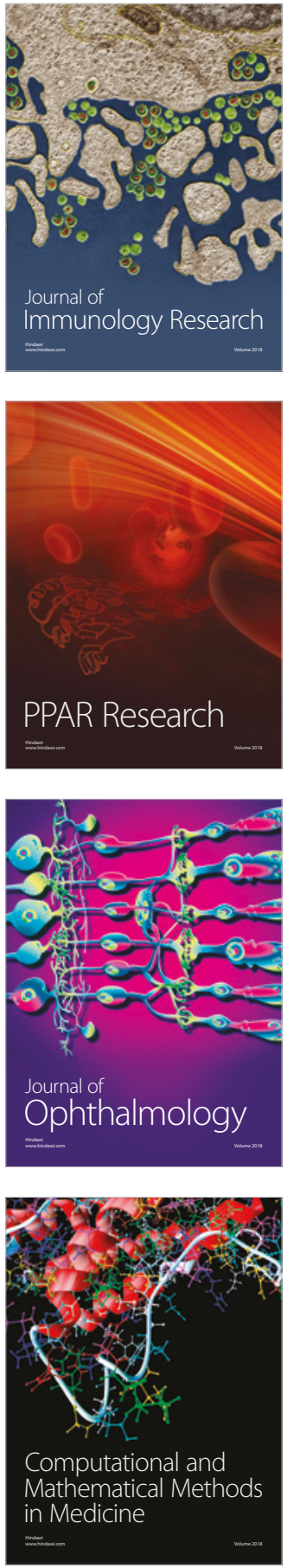

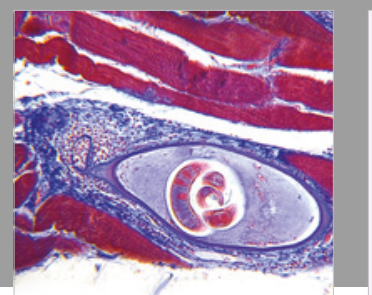

Gastroenterology Research and Practice

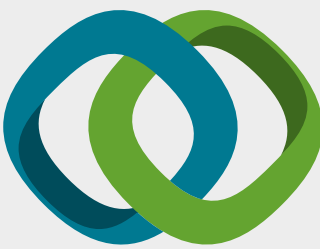

\section{Hindawi}

Submit your manuscripts at

www.hindawi.com
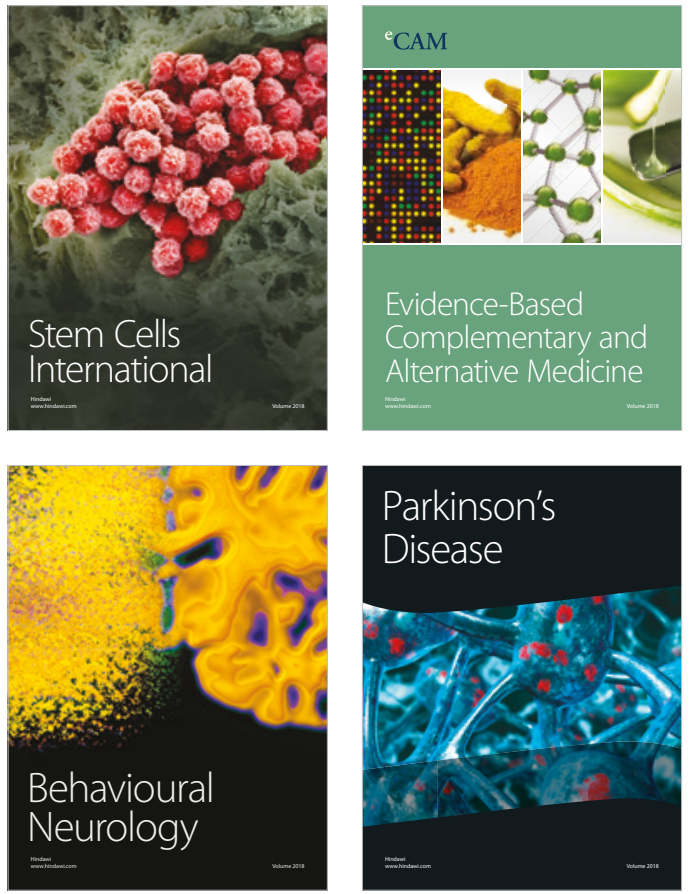

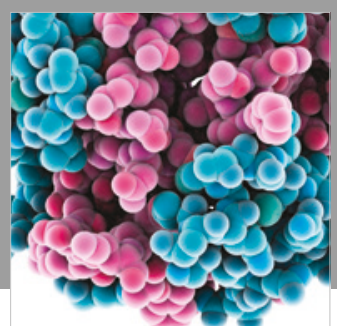

ournal of

Diabetes Research

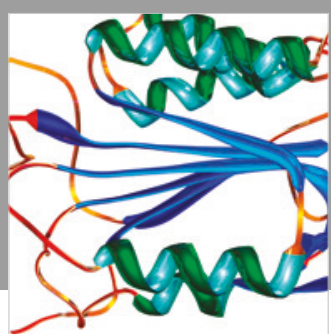

Disease Markers
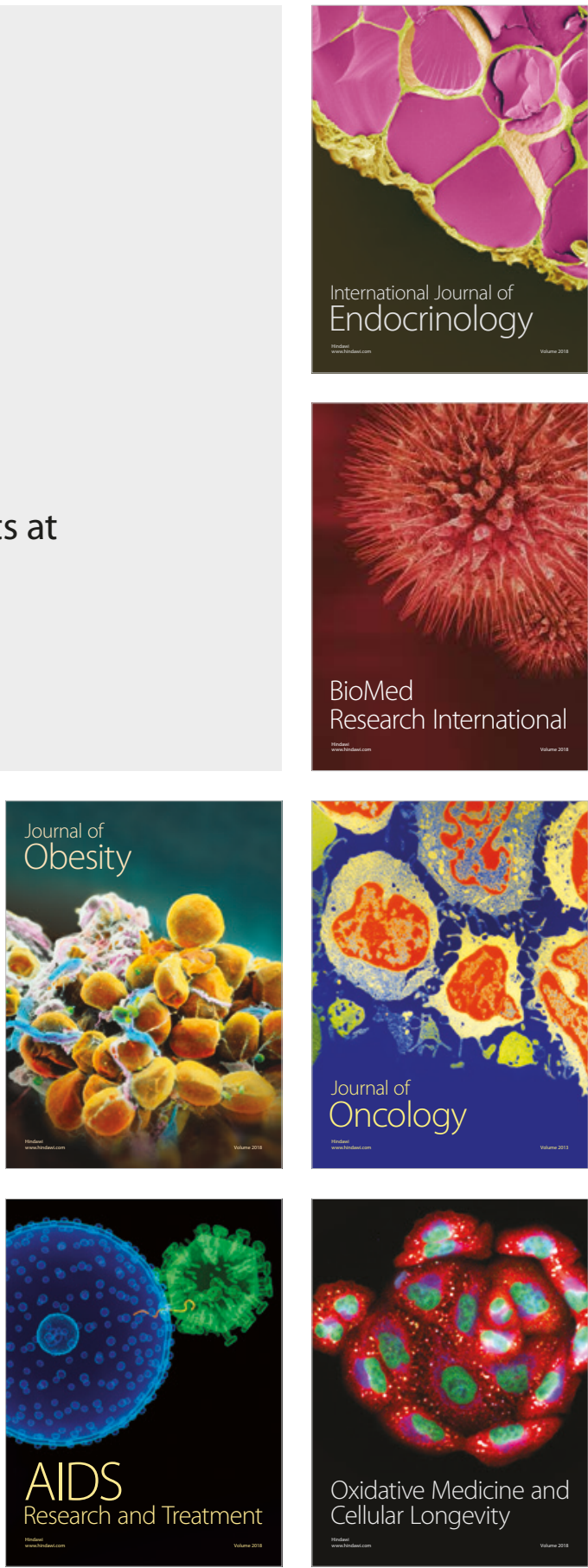